\title{
The Critical Phase Changes of a Forward-scattered Light in a Nonpolar Binary Liquid Mixture
}

\author{
Dong J. Lee \\ Deparment of Chemistrv. Pukiong National Cniversitn, Nam-Gu. Busan 608-737, Korea \\ Received June 5. 2003
}

Key Words : Azimuth Ellipticity. Fisher correlation function

In a previous paper ${ }^{3}$ the author and his coworkers have obtained analytic results for the phase changes of the forward-scattered light in a nonpolar chiral fluid. when the incident light is contpletely linearly polarized above (or below) the horizontal at $45^{\circ}$. using the formalism given by Barron $^{2}$ and the theory for the dielectric tensor developed by Lee and $\mathrm{Kim}^{3}{ }^{3}$ The niethod is so effective that it enables us to calculate phase changes of the forward-scattered light in the critical region with the aid of Ornstein-Zernike theory of the correlation function of density fluctuations. Then, the method was extended to a binary liquid mixture composed of a chiral solute and an achiral solvent. ${ }^{+}$The mixture is more suitable for experimental verification than the pure fluid. The basic difference with the latter is that there is one more extra variable in the mixture, i.e., the concentration fluctuations. The results for the azimuth and ellipticity changes were extended in the critical region that the Fisher theory holds. ${ }^{\text {: }}$

The purpose of the present paper is to discuss the phase changes in the critical region of a nonpolar binary liquid mixture, using the Fisher form for the correlation function of concentration fluctuations. ${ }^{6,7}$ The explicit results are given without the detailed procedures. which were derived in the previous papers. ${ }^{1,4}$

\section{Results and Discussion}

The correlation function to be used is. taking the most dominant term ${ }^{6.7}$

$$
S_{2}(\vec{k})=\frac{k_{B} T \kappa}{\left(1+\xi^{2} k^{2}\right)^{1-\frac{\eta}{2}}} .
$$

where all the notations in this paper are referred to ref. 4 except the critical exponent for correlation function of concentration fluctuations, n. Substituting Eq. (1) into Eqs. (19) of ref. 4 and using the similar procedure as that in ref. 4 . we may calculate the azimuth change under the condition of $\xi>a$ and ellipticity change.

(A) The ellipticity change

The ellipticity change is given as

$$
\Delta \eta=\frac{\omega l}{4} \operatorname{Re}\left(\gamma_{y}^{\prime}-\gamma_{y:}^{\prime}\right)=\frac{\omega l}{2} \operatorname{Re} \gamma_{n}^{\prime}=-A_{\eta} \omega^{\prime} l[\Delta \eta]^{\prime},
$$

where

$$
\begin{gathered}
A_{c}=\frac{1}{24 \pi}\left[\frac{1}{3}\left(\varepsilon_{0}+2\right)\left(\varepsilon_{0}-1\right)\right]^{2} \bar{\alpha}_{0}^{1} \bar{\beta}_{0}^{1} \rho_{0}^{2}\left(\frac{k_{B} T \kappa}{\xi^{2}}\right) \\
{[\Delta \eta]^{\prime}=\left[3-\left(\bar{\alpha}_{0}^{\prime \prime}+\bar{x}_{1}^{0} \bar{\alpha}_{0}^{\prime}\right) \rho_{0}\right]\left[\frac{1}{\eta(2+\eta)(4+\eta) p^{4}}\right.} \\
\left.\quad \times\left\{\left(1+4 p^{2}\right)^{2}\left[1-\left(1+4 p^{2}\right)^{\frac{\eta}{2}}\right]-2 \eta\left[1+(6+\eta) p^{2}\right]\right\}\right] \\
+\frac{2 \bar{x}_{1}^{0} \bar{\alpha}_{0}^{1} \rho_{0}}{\left(\varepsilon_{0}+2\right) \eta(2+\eta) p^{2}}\left[1+2 p^{2}\left[1-\left(1+4 p^{2}\right)^{\frac{\eta}{2}}\right]\right. \\
+p^{2}\left[1-\left(1+4 p^{2}\right)^{\frac{1}{2}}\right]+\frac{\varepsilon_{0}+2}{4 \varepsilon_{0}}(2+\eta) p^{2} \\
\left.\left\{1-\left(1+4 p^{2}\right)^{\frac{\eta}{2}}-\frac{2 \eta p^{2}}{1+4 p^{2}}\left(1+4 p^{2}\right)^{\frac{\eta I}{2}}\right\}\right]+0(t)
\end{gathered}
$$

Using the following relation for a positive quantity $x$

$$
\lim _{n \rightarrow i n} \frac{1}{\eta}\left(x^{\eta}-1\right)=\ln x \text {. }
$$

it can be easily seen that as $\eta \rightarrow 0$, the above result reduces to that of Eq. (23) in ref. 4. The above result is so complicated that let us consider two limiting cases. If the system is in the critical region far from the critical point. the ellipticity change becomes

$$
\begin{aligned}
& \Delta \eta(\omega) \sim-\frac{\varepsilon_{0}}{6 \pi}\left[\frac{1}{3}\left(\varepsilon_{0}+2\right)\right]^{2}\left(\frac{3}{\varepsilon_{0}+2}-\frac{1}{3} \bar{x}_{1}^{0} \bar{\alpha}_{0}^{1} \rho_{0}\right) \\
& \times \bar{\alpha}_{0}^{\mid} \bar{\beta}_{0}^{\mid} \rho_{i j}^{2} k_{R} T \kappa \omega^{5} l . \quad \text { if } p \ll 1
\end{aligned}
$$

The above result. which is just the same as that in ref. 4 . is proportional to and diverges as the isothernal compressibility coefficient. becomes divergent. When the system is extremely close to the critical point, we have

$$
\begin{aligned}
& \Delta \eta=A_{i}\left[\frac{27}{2\left(\varepsilon_{0}+2\right)}-\bar{x}_{1}^{0} \bar{\alpha}_{0}^{\prime} \rho_{0}\left(\frac{1}{2}+\frac{\varepsilon_{0}+2}{4 \varepsilon_{0}}\right)\right. \\
&\left.-\left(\frac{18}{\varepsilon_{0}+2}+\bar{x}_{1}^{j} \bar{\alpha}_{0}^{\prime} \rho_{0 j} \frac{\varepsilon_{0 j}+2}{2 \varepsilon_{0}}\right) \times \frac{1}{\eta}\left\{1-(2 p)^{\eta}\right\}\right] \omega^{3} l . \\
& \text { if } p>>1 .
\end{aligned}
$$

The ellipticity change in the extreme critical point behaves $\sim \omega^{3}\left(n_{0}, \xi \omega\right)^{n}$. whereas it in a fluid satisfying the Ornstein- 
Zernike theory shows logarithmic divergence. $\sim \omega)^{3} \ln \left(n_{0} \xi(\omega)\right.$

(B) The azimuth change. $\Delta \theta(\omega)$ in the critical region may be separated into three parts

$$
\begin{aligned}
\Delta \theta(\omega) & =\frac{\omega l}{4} \operatorname{Re}\left(\gamma_{y}^{\prime}-\gamma_{i}^{\prime}\right) \\
& =\Delta \theta_{1}(\omega)+\Delta \theta_{2}{ }^{\prime}(\omega)+\Delta \theta_{3}{ }^{\prime}(\omega)
\end{aligned}
$$

The first term $\Delta \theta_{1}(\omega)$ is due to the molecular contribution given by

$$
\Delta \theta_{1}(\omega)=-\frac{3 n_{0}}{\varepsilon_{u}+2} \bar{\beta}_{\omega}^{\prime} \rho_{\omega} \omega \omega^{2} l
$$

The second term $\Delta \theta_{2}{ }^{\prime}(\omega)$ is

$$
\begin{aligned}
& \Delta \theta_{2}^{\prime}(\omega) \simeq-\frac{1}{48 \pi}\left(\frac{3}{\varepsilon_{0}+2}\right)\left(\varepsilon_{\dot{0}}-1\right)\left[1-\frac{1}{3}\left(\bar{\alpha}_{\dot{u}}^{\ddot{u}}+\bar{x}_{1}^{\dot{v}} \bar{\alpha}_{0}^{\prime}\right) \rho_{0}\right] \\
& \times \bar{\alpha}_{0}^{\prime} \bar{\beta}_{0}^{\prime} \rho_{i v}^{2}\left(\frac{k_{B} T \kappa}{\xi^{2}}\right)\left(\begin{array}{l}
\xi \\
a
\end{array}\right)^{1-\eta} \Psi\left(\eta \cdot \bar{\alpha}_{0}^{0} \rho_{0}\right) \omega^{2} l .
\end{aligned}
$$

with

where

$$
\Psi\left(\eta \cdot \bar{\alpha}_{0}^{n} \rho_{0}\right)=\int d x \frac{x^{n} \Omega(x)^{2}}{1+\Omega(x)}
$$

$$
\begin{aligned}
\Omega(x) & =3 \bar{\alpha}_{i j}^{\prime} \rho_{0} f(x)\left[1-\bar{\alpha}_{i}^{\prime} \rho_{0} f(x)\right]^{-1} ; \\
f(x) & =x^{-3} \sin x-x^{-2} \cos x
\end{aligned}
$$

When $\eta=0$. the approximate value of the function is $3 \pi / 5\left(3 \bar{\alpha}_{i, g}^{g} \rho_{0} / 3+2 \bar{\alpha}_{i}^{g} \rho_{0}\right)^{2}$ and is of order 1 for $0 \leq \bar{\alpha}_{i v}^{0} \rho_{0}$ $\leq 1.5 . \Psi\left(\eta \cdot \bar{\alpha}_{0}^{\prime} \rho_{0}\right)$ is also of the order $l$ for $0 \leq \bar{\alpha}_{0}^{0} \rho_{0} \leq 1.5$ and $0<\eta<1$. The last term in Eq. (8) is

$$
\Delta \theta_{3}^{\prime}(\omega)=\Delta \theta_{3}(\omega)\left(1+p^{2}\right)^{\frac{1}{2}} .
$$

where $\Delta \theta_{3}(\omega)$ is given in Eq. (34) of ref. 4. As discussed in ref. 4. $\Delta \theta_{2}^{\prime}(\omega)$ is dominant over $\Delta \theta_{3}^{\prime}(\omega)$ in the case that the system is far from the critical point. As the system approaches to the critical point. $\Delta \theta_{3}^{\prime}(\omega)$ becomes more important. If $\mathrm{I}<<p<|t|^{-1}$, we have

$$
\Delta \theta_{i}^{\prime}(\omega)=-\left(\frac{\varepsilon_{0}+2}{3}\right)^{2}\left(\bar{\alpha}_{u}^{l} \rho_{0}\right)\left(k_{B} T \kappa \xi^{2-\eta}\right) n_{0}^{\eta} \omega^{\hat{2} \eta \eta}|| \ell \mid .
$$

The magnitude of $\Delta \theta^{\prime}(\omega)$ may become comparable to or larger than $\Delta \theta_{2}^{\prime}(\omega)$. It is due to the indirect coupling between two fluctuating parts of dipole densities via the equilibrium part of quadrupole density in the middle. In the extreme case where $p|t| \gg>1$. we have

$$
\Delta \theta_{3}(\omega) \simeq-\left(\frac{\varepsilon_{0}+2}{3}\right)^{2}\left(\bar{\alpha}_{0}^{1} \rho_{0}\right)^{2}\left(k_{B} T \kappa / 32 \pi \eta_{0}^{1-\pi} \xi^{2-\pi}\right) \omega^{2+\pi} l .
$$

The above result is independent of $\bar{\beta}_{0}^{\prime \prime}$. Its magnitude may become comparable to or larger than the term due to the molecular contribution. $\Delta \theta_{1}(\omega)$, depending on the magnitude of $k_{R} T \kappa / \xi^{2-\eta}$.

Let us conclude some important results:

(1). The ellipticity change. where the system is far from the critical point, is proportional to $\omega^{*}$ and diverges as the isothermal compressibility coefficient becomes divergent.

(2). When the system is extremely close to the critical point. the ellipticity change behaves $\sim \omega^{3}\left(n_{i} \xi \xi(0)^{n}\right.$, whereas it in a fluid satisfying the Onnstein-Zennike theory shows logarithmic divergence. $\sim\left(\omega^{2} \ln \left(n_{i} \xi \xi(\omega)\right.\right.$.

(3). The molecular term plays the most dominant role on the azimuth clange of the scattered light in the case that the system is far from the critical point.

(4). As the system approaches to the critical point. the effect of density fluctuations on the azimuth clange becomes important. In the extreme close to the critical point, the effect is at least comparable to the molecular contribution.

(5). It is known that the value of exponent $\eta$ is between 0 $0.1{ }^{6.7}$ In the extreme critical region we may experimentally measure the exponent by observing the dependence of the azimuth change on the frequency of the light.

\section{References}

1. Kim. Y; Kim, K. R.; Kang, Y. S.: Lee, D. J. Chem. Phus. 2002. 283.1573.

2. Barron. L. D. Molecular Light Scattering and Optical Activity: Cambridge University Press: Cambridge. 1982.

3. Kim. S. K.; Lee, D. J. J. Chen. Phys 1996. 105. 5341.

4. Kim. K. R; Kang. Y. S, Lee, D. J. Bull Koman Chem Soc. 2002. 23. 1753 .

5. Kiml. K. R.: Lee. D. J. in press in Chem. Plys. Letters.

6. Fisher. M. E. J. Math Phys 1964.5.944.

7. Stanley. H. E. Introdiction to Phase Transition and Critical Phenontena: Claredon: Oxford. 1971: p 105. 